\title{
Lung tissue distribution of drugs as a key factor for COVID-19 treatment
}

\author{
Yan WANG ${ }^{1}$ and Lei Chen ${ }^{2}$ \\ ${ }^{1}$ Shenzhen Institutes of Advanced Technology Chinese Academy of Sciences \\ ${ }^{2}$ Rutgers The State University of New Jersey
}

April 28, 2020

\begin{abstract}
Lopinavir combined with ritonavir were reported to benefit the patients with SARS by reducing the viral loads. However, in the latest clinical trials, no benefit was observed with lopinavir-ritonavir treatment beyond standard care in patients with COVID19. We comment here that this disappointed result of clinical trial might result from the low volume of the lung distribution of lopinavir. The major reasons were listed below: 1) The binding affinity of ACE2 with SARS-CoV-2 spike protein is $~ 10-$ to 20-fold higher than the binding affinity of ACE2 with SARS-CoV spike protein, indicating that SARS-CoV-2 can enter AT2 cells in lung much easier than SARS-CoV. Therefore, the viral loads of SARS-CoV-2 might be much higher than viral loads of SARS-CoV in the lung tissue. 2) The concentration of lopinavir in the lung tissue was $1.18 \mu \mathrm{g}$ equiv/ml in rats. The low volume of the lung distribution of lopinavir might not be enough to inhibit the coronavirus replication due to the high viral loads in the lung tissue. 3) In contrast, the concentration of chloroquine in the lung tissue was much higher $(30.76 \pm 0.85 \mu \mathrm{g}$ equiv $/ \mathrm{ml}$ ) in rats, which might lead to its clinical and virologic benefits in the treatment of COVID-19 patients. Together, we proposed here that anti-SARS-CoV-2 drug repurposing studies should pay more attentions to the lung tissue distribution of antiviral drugs. The efficacy of antiviral drugs might depend on their lung tissue distributions
\end{abstract}

\section{Lung tissue distribution of drugs as a key factor for COVID-19 treatment}

Yan Wang ${ }^{1, *}$, Lei Chen ${ }^{2}$

${ }^{1}$ Center for Translation Medicine Research and Development, Shenzhen Institute of Advanced Technology, Chinese Academy of Sciences, Shenzhen 518055, P.R. China

${ }^{2}$ Department of Genetics, Human Genetics Institute of New Jersey, Rutgers University, Piscataway, NJ 08854, USA

*Correspondence: Dr. Yan Wang, Center for Translation Medicine Research and Development, Shenzhen Institute of Advanced Technology, Chinese Academy of Sciences, Shenzhen 518055, P.R. China; 86755-2641 7985; E-mail: yan.wang@siat.ac.cn

Over 72,000 people around the world was killed by Coronavirus Disease 2019 (COVID-19). COVID-19 is caused by severe acute respiratory syndrome coronavirus 2 (SARS-CoV-2). It is impossible to create novel drugs against the coronavirus in very short time, as it often takes years; therefore, the best strategy is to find new antiviral uses from approved drugs [1]. Not surprisingly, SARS-CoV-2 shares a highly similar viral genome sequence with SARS-CoV [2], suggesting that the effective treatments for SARS may also work for COVID-19 treatment.

Lopinavir, a human immunodeficiency virus type 1 (HIV-1) protease inhibitor, showed a good inhibitory effect on SARS-CoV replication in cell-based assays. In clinical trials, lopinavir combined with ritonavir benefited the patients with SARS by reducing the viral loads [3]. According to molecular docking and 
dynamics analysis, lopinavir has been identified as a main protease inhibitor of SARS-CoV, and approved for inhibiting the SARS-CoV replication [4]. Recent docking simulation studies showed that lopinavir can also directly bind to catalytic pocket of SARS-CoV-2 main protease, indicating its potential to reduce the viral loads in patients with COVID-19 [5]. However, in clinical trials, no benefit was observed with lopinavirritonavir treatment beyond standard care in patients with COVID-19 [6].

Both SARS-CoV and SARS-CoV-2 can attach to angiotensin-converting enzyme 2 (ACE2) and then enter host cells [2]. Given that ACE2 is highly expressed in AT2 cells in lung [7], lung becomes a major organ under the coronavirus attack. Interestingly, ACE2 bound to the SARS-CoV-2 spike protein with $15 \mathrm{nM}$ affinity, which is 10- to 20-fold higher than ACE2 binding to SARS-CoV spike protein [8]. It is indicated that SARS-CoV-2 can enter AT2 cells in lung much easier comparing with SARS-CoV. The viral loads of SARS-CoV-2, in turn, might be much higher than viral loads of SARS-CoV in the lung tissue. Therefore, the anti-SARS-CoV-2 drugs should target against the lung rather than other tissues.

In a previous study, the tissue distribution of isotope-labeled lopinavir was examined in rats. The peak radioactivity levels in plasma were achieved at $4 \mathrm{~h}$ post-administration. At $4 \mathrm{~h}$ after administration (10 $\mathrm{mg} / \mathrm{kg}$ ), liver $(52.24 \mu \mathrm{g}$ equiv $/ \mathrm{ml})$, adrenals $(4.80 \mu \mathrm{g}$ equiv $/ \mathrm{ml})$, and thyroid $(4.41 \mu \mathrm{g}$ equiv $/ \mathrm{ml})$ exhibited greater radioactivity levels than plasma. The lung $(1.18 \mu \mathrm{g}$ equiv/ml $)$ exhibited less radioactivity levels than plasma, indicating that the distribution of lopinavir in the lung tissue is relatively low [9]. We guess the concentration of lopinavir in the lung is too low to inhibit SARS-CoV-2 replication well. It might explain why lopinavir did not benefit the patients with COVID-19.

Unlike lopinavir, chloroquine exhibited clinical and virologic benefits in the treatment of COVID-19 patients, including improving lung image findings and reducing viral loads. In preclinical studies, chloroquine showed a strong inhibitory effect on SARS-CoV-2 replication in cell-based assays $\left(\mathrm{EC}_{50}=1.13 \mu \mathrm{M}\right)[10]$. Lung is one of the major target tissues as evidenced in tissue distribution studies of oral administration of chloroquine in rats. After an oral administration of ${ }^{14} \mathrm{C}$-chloroquine $(20 \mathrm{mg} / \mathrm{kg})$ to albino and pigmented rats, the lung tissue concentrations were similar $(30.76 \pm 0.85$ and $34.76 \pm 1.56 \mu \mathrm{g}$ equiv/ml, respectively) [11]. In a 32 -weeks treatment $(16.8 \mathrm{mg} / \mathrm{kg} /$ day $)$, the lung tissue concentrations of chloroquine were $51.7 \pm 3.1$ and 104 $\pm 7.0 \mu \mathrm{g} / \mathrm{mg}$ in male and female rats, respectively [12]. Both these two pharmacokinetics studies showed that the distribution of chloroquine is high in the lung distribution. We believed that chloroquine can take an advantage of the high volume of the lung distribution to inhibit the viral replication in the lung.

Together, we proposed that anti-SARS-CoV-2 drug repurposing studies should pay more attentions to the lung tissue distribution of antiviral drugs. The low volume of the lung distribution of antiviral drugs might not be enough to inhibit the coronavirus replication due to the high viral loads in the lung tissue. Among the anti-SARS-CoV-2 drugs in the clinical trials, hydroxychloroquine is likely to be a promising drug that benefit COVID-19 patients because of its high volume of the lung distribution. In a 32-weeks treatment $(19.4 \mathrm{mg} / \mathrm{kg} /$ day), the lung tissue concentration of hydroxychloroquine is $55.7 \pm 3.4 \mu \mathrm{g} / \mathrm{mg}$ [12]. So far, the most potent inhibitor of SARS-CoV-2 in vitro is remdesivir $\left(\mathrm{EC}_{50}=0.77 \mu \mathrm{M}\right)$ [10], which lacks of the tissue distribution data in public. Although remdesivir was reported to reduce MERS-CoV viral lung loads in animals [13], we are eager to see its lung tissue distribution data. It would help to choose an appropriate dosing and route of administration of remdesivir.

\section{Reference}

[1] Charlotte H, Coronavirus puts drug repurposing on the fast track. Nature Biotechnology News, doi: 10.1038/d41587-020-00003-1

[2] Wan Y, et al. Receptor Recognition by the Novel Coronavirus from Wuhan: an Analysis Based on Decade-Long Structural Studies of SARS Coronavirus. J Virol. 2020;94(7). pii: e00127-20.

[3] Stockman LJ, et al. SARS: systematic review of treatment effects. PLoS Med. 2006;3(9):e343.

[4] Nukoolkarn V, et al. Molecular dynamic simulations analysis of ritonavir and lopinavir as SARS-CoV 3CL(pro) inhibitors. J Theor Biol. 2008;254(4):861-7. 
[5] Alessandro C. Virtual screening of an FDA approved drugs database on two COVID-19 coronavirus proteins. DOI: 10.26434/chemrxiv.11847381.v1

[6] B Cao, et al. A Trial of Lopinavir-Ritonavir in Adults Hospitalized with Severe Covid-19. N Engl J Med. 2020. doi: 10.1056/NEJMoa2001282.

[7] Xu, H., et al. High expression of ACE2 receptor of 2019-nCoV on the epithelial cells of oral mucosa. Int J Oral Sci, 2020; https://doi.org/10.1038/s41368-020-0074-x

[8] Wrapp D, et al. Cryo-EM structure of the 2019-nCoV spike in the prefusion conformation. Science. 2020; 367: $1260-1263$

[9] Denissen JF, et al. Metabolism and disposition of the HIV-1 protease inhibitor ritonavir (ABT-538) in rats, dogs, and humans. Drug Metab Dispos.1997;25(4):489-501.

[10] Wang $\mathrm{M}$, et al. Remdesivir and chloroquine effectively inhibit the recently emerged novel coronavirus (2019-nCoV) in vitro. Cell Res. 2020; 30(3):269-271.

[11] Ono C, et al. Absorption, distribution and excretion of 14C-chloroquine after single oral administration in albino and pigmented rats: binding characteristics of chloroquine-related radioactivity to melanin in-vivo. J Pharm Pharmacol. 2003;55(12):1647-54.

[12] McChesney EW, et al. Tissue distribution of chloroquine, hydroxychloroquine, and desethylchloroquine in the rat. Toxicol Appl Pharmacol.1967;10(3):501-13.

[13] de Wit E, et al. Prophylactic and therapeutic remdesivir (GS-5734) treatment in the rhesus macaque model of MERS-CoV infection. Proc Natl Acad Sci U S A. 2020;117(12):6771-6776. 